\title{
WHEN CAN GRAVITATIONAL-WAVE OBSERVATIONS DISTINGUISH BETWEEN BLACK HOLES AND NEUTRON STARS?
}

\author{
Mark Hannam $^{1,2}$, Duncan A. Brown ${ }^{2,3}$, Stephen Fairhurst ${ }^{1,2}$, Chris L. Fryer ${ }^{2,4}$, and Ian W. Harry ${ }^{2,3}$ \\ ${ }^{1}$ School of Physics and Astronomy, Cardiff University, Cardiff, UK \\ ${ }^{2}$ Kavli Institute of Theoretical Physics, UC Santa Barbara, CA, USA \\ ${ }^{3}$ Department of Physics, Syracuse University, Syracuse, NY, USA \\ ${ }^{4}$ Computational Computer Science Division, Los Alamos National Laboratory, Los Alamos, NM, USA \\ Received 2013 January 26; accepted 2013 February 21; published 2013 March 8
}

\begin{abstract}
Gravitational-wave observations of compact binaries have the potential to uncover the distribution of masses and spins of black holes and neutron stars in the universe. The binary components' physical parameters can be inferred from their effect on the phasing of the gravitational-wave signal, but a partial degeneracy between the components' mass ratio and their spins limits our ability to measure the individual component masses. At the typical signal amplitudes expected by the Advanced Laser Interferometer Gravitational-wave Observatory (signal-to-noise ratios between 10 and 20), we show that it will in many cases be difficult to distinguish whether the components are neutron stars or black holes. We identify when the masses of the binary components could be unambiguously measured outside the range of current observations: a system with a chirp mass $\mathcal{M} \leqslant 0.871 M_{\odot}$ would unambiguously contain the smallest-mass neutron star observed, and a system with $\mathcal{M} \geqslant 2.786 M_{\odot}$ must contain a black hole. However, additional information would be needed to distinguish between a binary containing two $1.35 M_{\odot}$ neutron stars and an exotic neutron-star-black-hole binary. We also identify those configurations that could be unambiguously identified as black hole binaries, and show how the observation of an electromagnetic counterpart to a neutron-star-black-hole binary could be used to constrain the black hole spin.
\end{abstract}

Key words: black hole physics - gravitational waves - stars: neutron

Online-only material: color figures

\section{INTRODUCTION}

The first observing runs of the Advanced Laser Interferometer Gravitational-wave Observatory (aLIGO) are expected in 2015 , with Advanced Virgo following on a similar schedule (Harry et al. 2010; Acernese et al. 2009). The primary source for these observatories is the coalescence of binaries containing black holes (BHs) and/or neutron stars (NSs), with predicted rates between a few and several hundred per year at detector design sensitivity (Abadie et al. 2010; Dominik et al. 2012). The components of these binaries are formed in supernovae when the core of a massive star collapses to a compact remnant, although the exact collapse mechanism remains unknown. Detailed knowledge of the mass distribution of BHs and NSs will provide vital clues to their formation as well as explore the equation of state (EOS) of nuclear matter at high densities.

Measuring the upper and lower limits of NS masses allows us to constrain the supernova engine and the nuclear physics of NS remnants (Lattimer \& Prakash 2011; Fryer et al. 2012). For example, the collapse of low-mass stars $\left(8-12 M_{\odot}\right)$ is believed to quickly produce explosions with very little mass accreted in a convective engine phase. The predicted mass of the compact remnant will be less than the Chandrasekhar mass by an amount that depends on the collapse model (Fryer et al. 1999; Kitaura et al. 2006; Dessart et al. 2007). If we can place an upper limit on the mass of a low-mass NS (in the 1.0-1.2 $M_{\odot}$ range), we can distinguish between current models, effectively using these low-mass systems to constrain the EOS and neutrino physics in core collapse. At higher masses, Ozel et al. (2010) and Farr et al. (2011) have argued that there is a gap between $\sim 2$ and $4 M_{\odot}$ where no compact objects exist. If true, the amount of material that falls back onto the newly formed compact remnant must be small, arguing against engine mechanisms that take a long time (more than $200 \mathrm{~ms}$ ) to develop (Belczynski et al. 2012). However, this mass gap may simply be an artifact of poor mass resolution of X-ray binaries and poor statistics (Kreidberg et al. 2012). BH mass distributions will allow us to explore the fall-back of material in a weak supernova explosion and $\mathrm{BH}$ masses in solar metallicity environments will provide clues to stellar mass loss. With an accurate BH mass distribution, we can study these open questions in stellar evolution.

The binary's gravitational-wave phasing depends at leading order on its chirp mass $\mathcal{M}=\left(m_{1} m_{2}\right)^{3 / 5}\left(m_{1}+m_{2}\right)^{-1 / 5}$, where $m_{1}$ and $m_{2}$ are the binary's component masses (Peters \& Mathews 1963); this quantity will be most accurately measured in a gravitational-wave detection. The mass ratio $\eta=$ $m_{1} m_{2} /\left(m_{1}+m_{2}\right)^{2}$ enters through higher order corrections and is less accurately measured; see e.g., Blanchet (2002). There is also a partial degeneracy between the mass ratio and the angular momentum $\chi_{1,2}=J_{1,2} / m_{1,2}^{2}$ of each compact object (the spin), which further limits our ability to measure the binary's component masses. Heuristically, this can be understood as follows: a binary with spins aligned with the orbital angular momentum will inspiral more slowly than a non-spinning system. Similarly, a binary of the same total mass but with more extreme mass ratio will inspiral more slowly. However, a binary with the same chirp mass but with more extreme mass ratio will inspiral more quickly. The effect on the waveform of decreasing $\eta$ can be mimicked by increasing the component spins.

We investigate the accuracy with which the component masses can be determined from gravitational-wave observations of binary-NS (BNS), NS-BH (NSBH), and binary-BH (BBH) systems, focusing on systems where the object's spins are aligned with the orbital angular momentum. Since the first 
signals detected by aLIGO are likely to have signal-to-noise ratios (S/Ns) close to the observable network threshold of $\sim 12$ (Abadie et al. 2012), we focus on signals with $\mathrm{S} / \mathrm{Ns}$ $10-20$, which will account for $\sim 80 \%$ of observations. For these $\mathrm{S} / \mathrm{Ns}$, we find that the mass-ratio-spin degeneracy will prevent us from accurately measuring component masses. We identify the region of the mass parameter space for which it will not be possible to determine whether the compact objects are BHs or NSs using gravitational-wave observations alone, when we can conclusively measure compact-object masses outside the currently observed limits, and show how the observation of an electromagnetic counterpart to an NSBH could be used to constrain the $\mathrm{BH}$ spin.

\section{PARAMETER ESTIMATION METHOD}

Fisher matrix methods show that the binary's chirp mass is recovered well by matched filtering, with accuracies of $\sim 0.01 \%$ for typical BNS systems in aLIGO (Finn \& Chernoff 1993; Arun et al. 2005). If we assume that the NSs are non-spinning $\eta$ can be measured to an accuracy of $\sim 1.3 \%$ (Arun et al. 2005). Estimates of the effect of the mass-ratio-spin degeneracy were first made by Cutler \& Flanagan (1994) and Poisson \& Will (1995) using the Fisher approach. The degeneracy between the mass ratio and the total effective spin $\chi=\left(m_{1} \chi_{1}+m_{2} \chi_{2}\right) /\left(m_{1}+m_{2}\right)$ degrades the ability to measure the mass ratio and hence the component masses. We go beyond these studies, using the method introduced in Baird et al. (2013), to equate a confidence interval with a region where the match between the signal and model waveforms exceeds a given threshold. We use this method to investigate parameter degeneracies for a wide range of binaries and interpret the expected measurement accuracy in the context of the astrophysical questions discussed above.

We model the waveforms with the TaylorF2 inspiral approximant (Sathyaprakash \& Dhurandhar 1991; Cutler \& Flanagan 1994; Droz et al. 1999) to leading order in amplitude and 3.5 post-Newtonian (PN) order in phase (Blanchet et al. 1995, 2002, 2004, 2005) with spin-orbit terms to 2.5PN order and spin terms to 2PN order (Kidder et al. 1993; Kidder 1995). For systems with total masses below $\sim 8 M_{\odot}$, our results with TaylorF2 are consistent with those from phenomenological BBH models that include the merger and ringdown (Ajith et al. 2011; Santamaria et al. 2010), calibrated against numerical-relativity waveforms with mass ratios up to 1:4 (Hannam et al. 2010). For the higher mass BBH results in Section 4 we use the full merger model. Throughout, we assume for simplicity that the component spins are aligned with the orbital angular momentum. In this case, the binary's distance, orientation, and sky location affect only the overall amplitude of the waveform, and we do not consider them here.

For two waveforms $h_{1}$ and $h_{2}$ the match is given by

$$
M=\max _{\Delta t, \Delta \phi} \frac{\left(h_{1} \mid h_{2}\right)}{\sqrt{\left(h_{1} \mid h_{1}\right)\left(h_{2} \mid h_{2}\right)}},
$$

where $(a \mid b)$ is the standard noise-weighted inner product

$$
(a \mid b)=4 \operatorname{Re} \int_{0}^{\infty} \frac{\tilde{a}(f) \tilde{b}^{*}(f)}{S_{n}(f)} d f .
$$

In all cases we use a noise sensitivity $S_{n}(f)$ corresponding to the zero-detuned high-power configuration of aLIGO (Shoemaker 2009) with a $15 \mathrm{~Hz}$ low frequency cutoff. We construct a $90 \%$ confidence region for a signal in the $\left(m_{1}, m_{2}, \chi\right)$ space, which

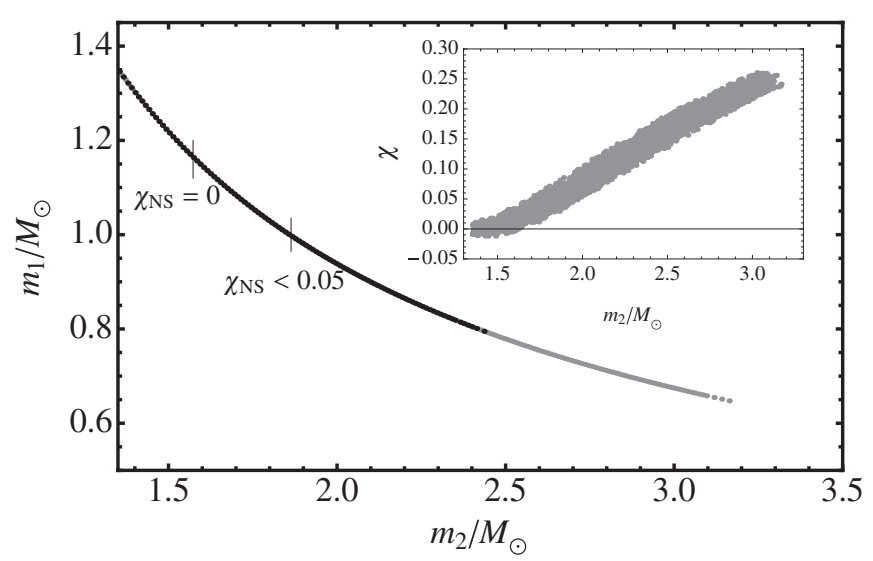

Figure 1. $90 \%$ confidence region around a $1.35-1.35 M_{\odot}$ BNS system at S/N 10 (gray) and S/N 20 (black) in the zero-detuned high-power configuration of aLIGO. The vertical bars indicate where the $\mathrm{S} / \mathrm{N} 10$ confidence region would be truncated if we restrict to non-spinning NSs, and if we restrict to NS spins less than 0.05 . The inset shows the total effective spin $\chi=$ $\left(m_{1} \chi_{1}+m_{2} \chi_{2}\right) /\left(m_{1}+m_{2}\right)$ of the waveforms, with respect to the mass of the larger body.

corresponds to the three-dimensional region where $M \geqslant 0.968$ (0.992) for an $\mathrm{S} / \mathrm{N}$ of 10 (20) (Baird et al. 2013). This method is more accurate at low $\mathrm{S} / \mathrm{Ns}$ than the Fisher matrix approach.

\section{NEUTRON-STAR BINARIES}

Observed NS masses currently lie between $1.0 \pm 0.10 M_{\odot}$ (Rawls et al. 2011) and $1.97 \pm 0.04 M_{\odot}$ (Demorest et al. 2010). General relativity and causality place a strict upper limit on the maximum NS mass of $3.2 M_{\odot}$ (Rhoades \& Ruffini 1974); the actual maximum mass is determined by the as yet unknown NS EOS. The observed masses of stars in double NS systems are narrower with a peak at $1.35 M_{\odot}$ and a width of $0.13 M_{\odot}$ (Kiziltan et al. 2010). We begin by considering a canonical BNS system with masses $m_{1}=m_{2}=1.35 M_{\odot}$ and no spins. Figure 1 shows the regions of the mass plane that are consistent with this source at $90 \%$ confidence for S/Ns 10 and 20. The component masses consistent with the signal lie roughly along a line of constant chirp mass. However, there is significant spread in the recovered component masses due to the degeneracy between $\eta$ and $\chi_{1,2}$ in the gravitational-wave phase evolution. Typically, the degeneracy persists over a range of 0.3 in $\chi$.

If we assume that the compact objects are non-spinning, the component masses are recovered in the ranges $1.15-1.35 M_{\odot}$ and 1.35-1.6 $M_{\odot}$. The observed spins of double NSs are low, with a minimum observed period of $22.70 \mathrm{~ms}$ for J0737-3039A (Burgay et al. 2003), i.e., $\chi \sim 0.02$, where the NS period can be related to the spin by approximately

$$
\chi=\left(\frac{2 \pi c I}{G m^{2}}\right)\left(\frac{1}{T}\right) \approx 0.4\left(\frac{1 \mathrm{~ms}}{T}\right) .
$$

If we constrain the NSs to have a spin $\chi \leqslant 0.05$, the range of consistent component masses extends to $1.0-1.35 M_{\odot}$ and 1.35-1.9 $M_{\odot}$. Therefore, at typical S/Ns, we would be unable to distinguish the canonical $1.35 M_{\odot}$ BNS from a more exotic BNS with $m_{1}=1.0 M_{\odot}, m_{2}=1.9 M_{\odot}$.

The fastest spinning pulsar (PSR J1748-2446ad) has a period of $1.4 \mathrm{~ms}$, or $\chi \sim 0.3$ (Hessels et al. 2006). NSs are considered unlikely to have a period less than $1 \mathrm{~ms}$ (Chakrabarty 2008), although breakup frequencies could be a factor of $\sim 2$ higher (Lo \& Lin 2011). If we allow for larger component spins, the region consistent with the canonical BNS system extends 


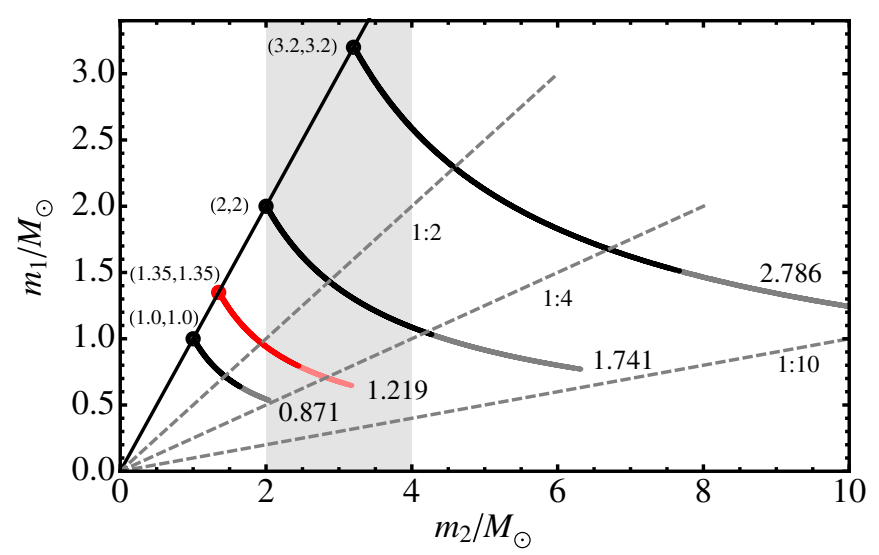

Figure 2. $90 \%$ confidence regions for a number of different non-spinning compact-binary configurations; see the text for interpretation. The number at the end of each confidence region is the chirp mass of the binary. The gray shaded region indicates the current observational mass gap.

(A color version of this figure is available in the online journal.)

to $0.65-1.35 M_{\odot}$ and $1.4-3.1 M_{\odot}$. The maximum total spin of binaries within the $90 \%$ confidence region at $\mathrm{S} / \mathrm{N} 10$ is $\sim 0.3$; see Figure 1 inset. Based on gravitational-wave observations alone, a binary of two $1.35 M_{\odot}$ NSs could not be definitively distinguished from a low-mass NS and a BH or NS in the mass gap, even at S/N 20. If the larger object is an NS its spin would be unusually large, but not impossible. To constrain the larger object's mass below $2 M_{\odot}$ without assumptions on the spins would require a signal with an $\mathrm{S} / \mathrm{N}$ of $\gtrsim 40$, expected for $\sim 2 \%$ of observations.

In Figure 2, we consider a family of equal mass, non-spinning BNS systems with component masses at the lowest $\left(1.0 M_{\odot}\right)$ and highest $\left(2.0 M_{\odot}\right)$ observed NS masses, and at the highest theoretical NS mass $\left(3.2 M_{\odot}\right)$. The $1.35 M_{\odot}$ binary is shown for reference, and the observed mass gap is indicated by the shaded region. Again, the $90 \%$ confidence regions follow lines of approximately constant chirp mass. We can see that for an exceptionally low or high mass binary, we will be able to identify at least one of the components as extraordinary. The observation of a BNS with a chirp mass $\mathcal{M} \leqslant 0.871 M_{\odot}$ would yield the unambiguous detection of a compact object with mass less than $1.0 M_{\odot}$. The less massive component could have a mass in the range $0.5-1.0 M_{\odot}$, but any mass in that range would challenge our current understanding of NSs and their formation in supernovae. A chirp mass $\mathcal{M}<1.045$ would yield an unambiguous detection of a $<1.2 M_{\odot}$ NS. This constraint would rule out many modern calculations of stellar collapse (Kitaura et al. 2006; Dessart et al. 2007) and provide a strong validation test for future calculations.

Similarly, the observation of a system with $\mathcal{M} \geqslant 1.741 M_{\odot}$ would indicate the detection of an NS of mass $\geqslant 2.0 M_{\odot}$ provided we can unambiguously identify both components as NSs. However, the observation of a BNS system with $m_{1}=$ $m_{2}=2.0 M_{\odot}$ is consistent with a binary containing an NS and a $\mathrm{BH}$ in the mass gap or an exotic, low-mass NS and a $\mathrm{BH}$. Although any configuration along this line is of interest, we cannot strongly constrain the component masses with gravitational-wave observations at low $\mathrm{S} / \mathrm{N}$. In particular, even if we assume that NSs are non-spinning and that the minimum $\mathrm{BH}$ mass is $3 M_{\odot}$, we cannot rule out the possibility that it is an NSBH system: our assumption would only remove the portion of the confidence interval with $2.4 M_{\odot} \leqslant m_{2} \leqslant 3.0 M_{\odot}$, and

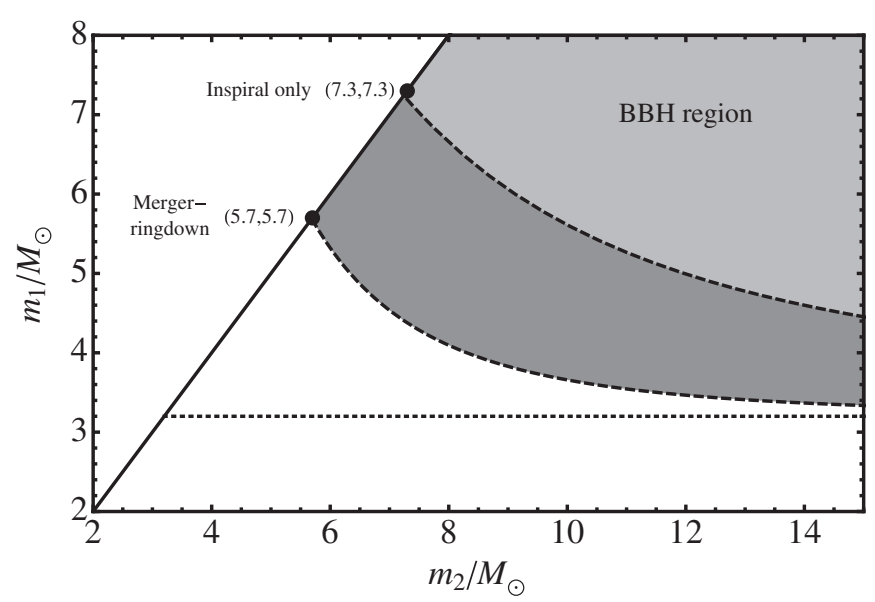

Figure 3. Shaded region indicates binaries would be unambiguously identified as BBH systems, assuming a maximum NS mass of $3.2 M_{\odot}$ (dotted line). The light gray region represents the most conservative estimate of this region, using an inspiral-only waveform model, while the additional darker region uses a full merger-ringdown model.

the observation would be of either a BNS system with wellconstrained masses or an NSBH.

Finally, we consider a binary with component masses at the upper end of the NS mass limit, above which we expect the components to be BHs. In this case, we could conclusively say that one of the components must be a $\mathrm{BH}$, although the degeneracy limits our ability to draw strong conclusions on the component masses. The binary could either be a BBH system with component masses in the mass gap or an NSBH system with masses consistent with previously observed compact objects.

Note that in all the cases we have considered, the equal-mass line provides a hard upper (lower) limit on $m_{1}\left(m_{2}\right)$.

Figure 2 shows that it will be difficult to explore the mass gap with single gravitational-wave observations at low S/Ns. To constrain one of the masses to lie within the mass gap, the system would need to be observed with an $\mathrm{S} / \mathrm{N}$ of greater than 30 ( $~ 5 \%$ of observations) ; in the case of the $m_{1}=m_{2}=2.0 M_{\odot}$ binary, the mass on one of the objects would then be constrained between $2 M_{\odot}$ and $3.5 M_{\odot}$, placing it directly within the mass gap.

\section{BINARY BLACK HOLES}

$\mathrm{BH}$ spins can vary between 0 and 1 , with observations of $\mathrm{X}$-ray binaries supporting the full range of values (Zhang et al. 1997). As before, the mass-ratio-spin degeneracy precludes precise measurement of the component masses. However, if we observed a $\mathrm{BBH}$ with $m_{1}=m_{2}=36 M_{\odot}$, corresponding to a binary with both components above $35 M_{\odot}$-the largest mass observed for a stellar-mass $\mathrm{BH}$ in an X-ray binary (Silverman \& Filippenko 2008) -we could conclude that one of the BHs has a mass of $36 M_{\odot}$ or higher, providing the first observational evidence for a stellar-mass $\mathrm{BH}$ above $35 M_{\odot}$. The same is true for any binary with the same or larger chirp mass, $\mathcal{M} \geqslant 31.34 M_{\odot}$.

A BBH will only be unambiguously identified if all binaries within the $90 \%$ confidence interval for the measured component masses are also BBH systems. The shaded region in Figure 3 shows the part of parameter space in which BBH systems could be identified as such at S/N 10, assuming a maximum NS mass of $3.2 M_{\odot}$. The upper curve shows the boundary of this region computed using the inspiral-only (TaylorF2) model. The lower 


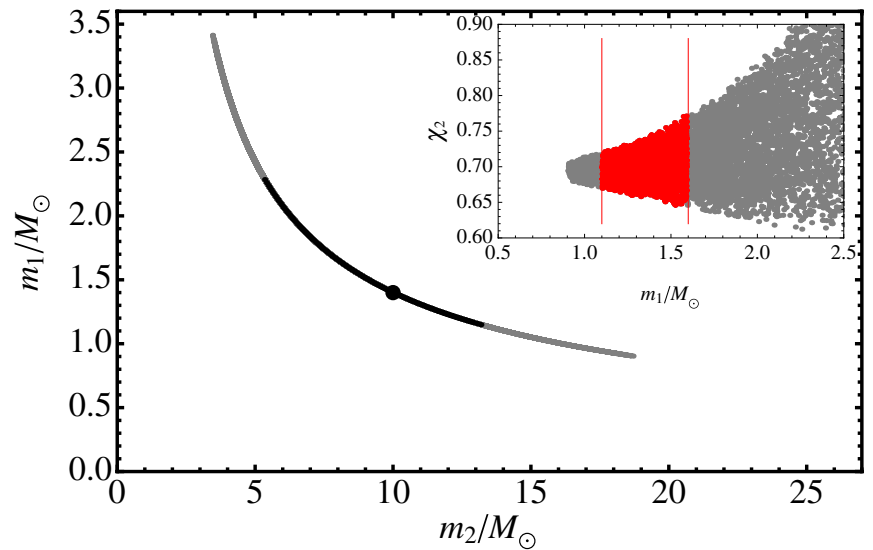

Figure 4. $90 \%$ confidence regions at S/Ns 10 and 20 for an NSBH system with masses $m_{1}=1.4 M_{\odot}$ and $m_{2}=10 M_{\odot}$, and BH spin $\chi_{2}=+0.7$. A GRB observation would allow an estimate of the BH mass of $m_{2}=11 \pm 3 M_{\odot}$, and $\chi_{2}=0.7 \pm 0.05$ (see the text).

(A color version of this figure is available in the online journal.)

curve is computed using a phenomenological inspiral-mergerringdown model (Santamaria et al. 2010). When the additional information from merger and ringdown is included, a larger region of the parameter space can be identified as a BBH system, illustrating the impact of merger-ringdown in these systems.

\section{ELECTROMAGNETIC COUNTERPARTS}

Figure 4 shows the $90 \%$ confidence regions for an NSBH, where the NS has mass $1.4 M_{\odot}$ and no spin, and the $\mathrm{BH}$ has $m_{2}=10 M_{\odot}$ with spin $\chi_{2}=0.7$ aligned with the orbital angular momentum. The uncertainty in both masses is large: this could be an NSBH system containing a BH between $6 M_{\odot}$ and $26 M_{\odot}$, or an equal-mass BBH system, with masses $m_{1}=$ $m_{2}=3.5 M_{\odot}$. The possible mass ratio of this system extends from $1: 1$ through to $1: 25$. Even at $\mathrm{S} / \mathrm{N} 20$ the mass ratio ranges from $1: 2.4$ up to $1: 11$. We note that the waveform models used here only include the waveform's dominant harmonic and do not include merger and ringdown (the phenomenological model is calibrated only up to mass ratio 1:4). The confidence region may significantly change if higher order or merger-ringdown effects are included, emphasizing the need for further waveform development in interpreting gravitational-wave observations as well as for detection.

The observation of a gamma-ray burst (GRB) or other electromagnetic signal would unambiguously identify one of the components as an NS and break the mass-ratio-spin degeneracy. If we accept that the mass of an NS in a binary is $1.35 \pm 0.25 M_{\odot}$ (Kiziltan et al. 2010), then the $\mathrm{BH}$ mass is restricted to $m_{2}=11 \pm 3 M_{\odot}$. The further assumption that the NS spin is below 0.4 restricts the possible values of the $\mathrm{BH}$ spin, allowing an estimate of $\chi_{2}=0.7 \pm 0.05$. This is shown in Figure 4 (inset), which shows the $90 \%$ confidence region restricted to the $\chi_{2}-m_{1}$ plane, and the region for which the NS mass is between $1.1 M_{\odot}$ and $1.6 M_{\odot}$. However, due to beaming only a fraction of gravitational-wave observations of BNS and NSBH systems are likely to be accompanied by GRB observations (Abadie et al. 2012a).

\section{CONCLUSIONS}

Although gravitational-wave observations will accurately measure the chirp mass $\mathcal{M}$ of binary mergers, a mass-ratio-spin degeneracy prevents the component masses and spins being measured accurately at low S/Ns. In many cases it will be difficult to determine whether the components of the binary are NSs or BHs. However, we have illustrated several cases where significant results can be inferred from gravitational-wave observations.

We illustrate several situations where the binary must contain (at least one) compact object that is more or less massive than anything observed to date; $\mathcal{M}<0.871 M_{\odot}$ indicates an NS smaller than $1 M_{\odot}, \mathcal{M}>1.741 M_{\odot}$ an NS larger than $2 M_{\odot}$ (if we can independently verify that it is a BNS system), and $\mathcal{M}>31.34 M_{\odot}$ a BH larger than $35 M_{\odot}$. Observations at $\mathrm{S} / \mathrm{Ns}$ higher than 30 will be required to clarify the existence of the mass gap. The observation of an electromagnetic counterpart will, in certain situations, allow us to identify the system as an NSBH and, if we know the distribution of NS masses, measure the $\mathrm{BH}$ mass and spin with high accuracy.

Our results are qualitatively robust, but the true confidence intervals may vary; they were generated by calculating waveform mismatches (Baird et al. 2013), rather than full parameterestimation methods (van der Sluys et al. 2008a, 2008b; Veitch \& Vecchio 2010; Feroz et al. 2009). In some cases inclusion of merger-ringdown, higher order PN corrections to spin effects, particularly at high mass ratios (Arun et al. 2009), and tidal effects will slightly alter the size of the error regions. These issues only serve to highlight the urgency of improved waveform models. The inclusion of precession and higher harmonics will modify our results, but we do not expect these to significantly break the degeneracy we discuss (Baird et al. 2013).

Our results highlight the significance of the mass-ratio-spin degeneracy in gravitational-wave observations of compact binaries. This needs to be explored further using more complete parameter-estimation methods, more accurate waveform models, and extended to include an exhaustive study of the parameter space. Results from a population of signals, rather than individual observations, should also be investigated. Understanding the uncertainties in masses and spin measurements will be essential to interpreting gravitational-wave observations.

We thank Harald Pfeiffer, Mark Scheel, and Patrick Sutton for helpful discussions. D.A.B. is supported by NSF award PHY-0847611 and an RCSA Cottrell Scholar award. S.F. is supported by the Royal Society. M.H. is supported by STFC grants ST/H008438/1 and ST/I001085/1. I.W.H. is supported by NSF grants PHY-0847611 and PHY-1205835. This work was funded in part under the auspices of the US Department of Energy, and supported by its contract W-7405-ENG-36 to Los Alamos National Laboratory. We thank the Kavli Institute for Theoretical Physics at UC-Santa Barbara, supported in part by NSF grant PHY11-25915, where this work was conceived.

\section{REFERENCES}

Abadie, J., Abbott, B. P., Abbott, R., et al. 2010, CQGra, 27, 173001

Abadie, J., Álvarez González, B., Amerio, S., et al. 2012, PhRvD, 85, 082002 Abadie, J., Watson, D. M., Kim, K. H., et al. 2012a, ApJ, 760, 12

Acernese, F., Alshourbagy, M., Antonucci, F., et al. 2009, Advanced Virgo Baseline Design [Virgo Techincal Document VIR-0027A-09; https://tds.egogw.it/q11?c=6589]

Ajith, P., Hannam, M., Husa, S., et al. 2011, PhRvL, 106, 241101

Arun, K., Buonanno, A., Faye, G., \& Ochsner, E. 2009, PhRvD, 79, 104023

Arun, K., Iyer, B. R., Sathyaprakash, B., \& Sundararajan, P. A. 2005, PhRvD, 71, 084008

Baird, E., Fairhurst, S., Hannam, M., \& Murphy, P. 2013, PhRvD, 87, 024035 Belczynski, K., Wiktorowicz, G., Fryer, C., Holz, D., \& Kalogera, V. 2012, ApJ, 757, 91

Blanchet, L. 2002, LRR, 5, 3 
Blanchet, L., Damour, T., Esposito-Farese, G., \& Iyer, B. R. 2004, PhRvL, 93, 091101

Blanchet, L., Damour, T., Iyer, B. R., Will, C. M., \& Wiseman, A. G. 1995, PhRvL, 74, 3515

Blanchet, L., Faye, G., Iyer, B. R., \& Joguet, B. 2002, PhRvD, 65, 061501, (erratum-ibid. D 71, 129902 [2005])

Blanchet, L., Faye, G., Iyer, B. R., \& Joguet, B. 2005, PhRvD, 71, 129902

Burgay, M., D’Amico, N., Possenti, A., et al. 2003, Natur, 426, 531

Chakrabarty, D. 2008, in AIP Conf. Ser., 1068, A Decade of Accreting Millisecond X-Ray Pulsars, ed. R. Wijnands, D. Altamirano, P. Soleri et al. (Melville, NY: AIP), 67

Cutler, C., \& Flanagan, E. E. 1994, PhRvD, 49, 2658

Demorest, P., Pennucci, T., Ransom, S., Roberts, M., \& Hessels, J. 2010, Natur, 467, 1081

Dessart, L., Burrows, A., Livne, E., \& Ott, C. D. 2007, ApJ, 669, 585

Dominik, M., Belczynski, K., Fryer, C., et al. 2012, ApJL, 759, L52

Droz, S., Knapp, D. J., Poisson, E., \& Owen, B. J. 1999, PhRvD, 59, 124016

Farr, W. M., Sravan, N., Cantrell, A., et al. 2011, ApJ, 741, 103

Feroz, F., Hobson, M. P., \& Bridges, M. 2009, MNRAS, 398, 1601

Finn, L. S., \& Chernoff, D. F. 1993, PhRvD, 47, 2198

Fryer, C., Benz, W., Herant, M., \& Colgate, S. A. 1999, ApJ, 516, 892

Fryer, C. L., Belczynski, K., Wiktorowicz, G., et al. 2012, ApJ, 749, 91

Hannam, M., Husa, S., Ohme, F., Muller, D., \& Bruegmann, B. 2010, PhRvD, 82,124008
Harry, G. M., \& LIGO Scientific Collaboration, 2010, CQGra, 27, 084006 Hessels, J. W., Ransom, S. M., Stairs, I. H., et al. 2006, Sci, 311, 1901 Kidder, L. E. 1995, PhRvD, 52, 821

Kidder, L. E., Will, C. M., \& Wiseman, A. G. 1993, PhRvD, 47, 4183

Kitaura, F., Janka, H.-T., \& Hillebrandt, W. 2006, A\&A, 450, 345

Kiziltan, B., Kottas, A., \& Thorsett, S. E. 2010, arXiv:1011.4291

Kreidberg, L., Bailyn, C. D., Farr, W. M., \& Kalogera, V. 2012, ApJ, 757,36

Lattimer, J. M., \& Prakash, M. 2011, in From Nuclei to Stars: Festschrift in Honor of Gerald E. Brown, ed. S. Lee (Singapore: World Scientific), 275

Lo, K.-W., \& Lin, L.-M. 2011, ApJ, 728, 12

Ozel, F., Psaltis, D., Narayan, R., \& McClintock, J. E. 2010, ApJ, 725, 1918

Peters, P. C., \& Mathews, J. 1963, PhRv, 131, 435

Poisson, E., \& Will, C. M. 1995, PhRvD, 52, 848

Rawls, M. L., Orosz, J. A., McClintock, J. E., et al. 2011, ApJ, 730, 25

Rhoades, C. E. J., \& Ruffini, R. 1974, PhRvL, 32, 324

Santamaria, L., Ohme, F., Ajith, P., et al. 2010, PhRvD, 82, 064016

Sathyaprakash, B., \& Dhurandhar, S. 1991, PhRvD, 44, 3819

Shoemaker, D. 2009, Advanced LIGO Reference Design [LIGO-M060056]

Silverman, J. M., \& Filippenko, A. V. 2008, ApJL, 678, L17

van der Sluys, M., Raymond, V., Mandel, I., et al. 2008a, CQGra, 25, 184011

van der Sluys, M. V., Röver, C., Stroeer, A., et al. 2008b, ApJL, 688, L61

Veitch, J., \& Vecchio, A. 2010, PhRvD, 81, 062003

Zhang, S., Cui, W., \& Chen, W. 1997, ApJL, 482, L155 